\title{
Transmission Eigenvalue Densities and Moments in Chaotic Cavities from Random Matrix Theory
}

\author{
PieRpaolo Vivo \\ School of Information Systems, Computing \& Mathematics \\ Brunel University \\ Uxbridge, Middlesex, UB8 3PH \\ (United Kingdom) \\ AND \\ EDOARDO VIVO \\ Università degli Studi di Parma \\ Dipartimento di Fisica Teorica \\ Viale G.P. Usberti n.7/A (Parco Area delle Scienze), Parma
}

(Italy)

\begin{abstract}
We point out that the transmission eigenvalue density and higher order correlation functions in chaotic cavities for an arbitrary number of incoming and outgoing leads $\left(N_{1}, N_{2}\right)$ are analytically known from the Jacobi ensemble of Random Matrix Theory. Using this result and a simple linear statistic, we give an exact and non-perturbative expression for moments of the form $\left\langle\lambda_{1}^{m}\right\rangle$ for $m>-\left|N_{1}-N_{2}\right|-1$ and $\beta=2$, thus improving the existing results in the literature. Secondly, we offer an independent derivation of the average density and higher order correlation functions for $\beta=2,4$ which does not make use of the orthogonal polynomials technique. This result may be relevant for an efficient numerical implementation avoiding determinants.
\end{abstract}

PACS numbers: 73.23.-b,73.50.Td,05.45.Mt,73.63.Kv

\section{Introduction}

Conductance in mesoscopic systems is currently a very active area of research, both from the theoretical and the experimental point of view. In the scattering theory framework [1][2], for the case of a chaotic cavity with $N_{1}$ and $N_{2}$ channels in each of the two attached leads, the fluctuations of the transmission eigenvalues of the conductor are effectively provided by a 
random matrix with appropriate symmetries [3][4]. More specifically, the Dyson index $\beta$ of the ensemble acquires the values 1 or 2 according to the presence or absence of time-reversal symmetry, or 4 in the case of spin-flip symmetry.

Several quantities of interest for the experiments, such as the conductance and the average shot noise, may be derived from the knowledge of the transmission eigenvalues $\left\{\lambda_{i}\right\}$. Those are defined as the singular values of a transmission matrix $t$, which in turn is a $N_{1} \times N_{2}$ off-diagonal block of a $\tilde{N} \times \tilde{N}$ unitary scattering matrix (where $\tilde{N}=N_{1}+N_{2}$ ) [5]. In the case of chaotic cavities considered below, $\left\{\lambda_{j}\right\}$ are correlated random variables between 0 and 1 .

Suppose that one is interested in computing the average shot noise $\langle P\rangle$, where:

$$
P=P_{0} \sum_{p=1}^{N} \lambda_{p}\left(1-\lambda_{p}\right), \quad N=\min \left(N_{1}, N_{2}\right),
$$

$P_{0}$ being a constant related to the physical properties of the conductor [5][6][7]. Until 2005, results for $\langle P\rangle$ were known only in the limiting cases $N_{1,2} \gg 1$ [2][8][9][10], $N_{1}=N_{2}=1$ [11] or few open channels [12]. Very recently, a compact form has been found for $\langle P\rangle / P_{0}$ using two different methods, based on a semiclassical expansion [13] and on recurrence relations for the Selberg integral [14] (see also [15] for an alternative derivation). The latter nicely exploits the remarkably simple expression for the joint probability density (jpd) of transmission eigenvalues:

$$
P_{\beta}\left(\lambda_{1}, \ldots, \lambda_{N}\right)=\mathcal{N}_{\beta}^{-1} \prod_{j<k}\left|\lambda_{j}-\lambda_{k}\right|^{\beta} \prod_{i=1}^{N} \lambda_{i}^{\frac{\beta}{2}\left(\left|N_{2}-N_{1}\right|+1\right)-1}, \quad 0 \leq \lambda_{j} \leq 1
$$

where the normalization constant is given by [14][16]:

$$
\mathcal{N}_{\beta}=\prod_{j=0}^{N-1} \frac{\Gamma\left(1+\frac{\beta}{2}+j \frac{\beta}{2}\right) \Gamma\left(\frac{\beta}{2}\left(\left|N_{2}-N_{1}\right|+1\right)+j \frac{\beta}{2}\right) \Gamma\left(1+j \frac{\beta}{2}\right)}{\Gamma\left(1+\frac{\beta}{2}\right) \Gamma\left(\frac{\beta}{2}\left(\left|N_{2}-N_{1}\right|+1\right)+1+(N+j-1) \frac{\beta}{2}\right)} .
$$

A few comments about (2) are in order. The jpd in (2) is stated in [2] without proof and attributed to Brouwer. A formal proof has been given (using three different methods) by Forrester [17] in 2006, where the author also highlighted the connection with the jpd of the Jacobi ensemble of random matrices [16][18]. In fact, one observes that the change of variables 
$y_{j}=1-2 \lambda_{j}$ brings (2) to the form:

$P_{\beta}\left(y_{1}, \ldots, y_{N}\right)=\tilde{\mathcal{N}}_{\beta}^{-1} \prod_{j<k}\left|y_{j}-y_{k}\right|^{\beta} \prod_{i=1}^{N}\left(1-y_{i}\right)^{\frac{\beta}{2}\left(\left|N_{2}-N_{1}\right|+1\right)-1}, \quad-1 \leq y_{j} \leq 1$,

allowing to use the machinery and results already known from Random Matrix Theory.

In particular, the average density of transmission eigenvalues:

$$
\rho_{\beta}\left(\lambda ; N_{1}, N_{2}\right)=\left\langle\sum_{i=1}^{N} \delta\left(\lambda-\lambda_{i}\right)\right\rangle=N \int_{[0,1]^{N-1}} d \lambda_{2} \ldots d \lambda_{N} P_{\beta}\left(\lambda, \lambda_{2} \ldots, \lambda_{N}\right)
$$

is of interest for computing linear statistics, i.e. observables of the form $\left\langle\operatorname{tr} f\left(t t^{\dagger}\right)\right\rangle$ :

$$
\left\langle\operatorname{tr} f\left(t t^{\dagger}\right)\right\rangle=\int_{0}^{1} d x \rho_{\beta}\left(x ; N_{1}, N_{2}\right) f(x) .
$$

The moments of the form $\left\langle\lambda_{1}^{m}\right\rangle$ for a real number $m$ can be computed in principle from the knowledge of the average density as:

$$
\left\langle\lambda_{1}^{m}\right\rangle=\int_{0}^{1} d x x^{m} \rho_{\beta}\left(x ; N_{1}, N_{2}\right),
$$

where the range for $m$ is constrained by the convergence of the integral. The first two moments are directly related to the normalized conductance $\left(G / G_{0}=\left\langle\lambda_{1}\right\rangle\right)$ thanks to the Landauer-Büttiker formula, and to the already mentioned shot noise $\left(P / P_{0}=\left\langle\lambda_{1}\right\rangle-\left\langle\lambda_{1}^{2}\right\rangle\right)$. A refined semiclassical treatment of the former can be found in [19].

Surprisingly, the connection with the Jacobi ensemble has not been fully appreciated so far, with the consequence that the average spectral density $\rho_{\beta}\left(\lambda ; N_{1}, N_{2}\right)$ for finite and arbitrary number of open channels $\left(N_{1}, N_{2}\right)$ is still deemed unknown (see e.g. [14][20]). On the other hand, the density is known in the above mentioned limiting cases [2][3][4][8][12][21].

In the mesoscopic literature the Jacobi ensemble is mentioned in the paper by Araújo and Macêdo [12], where the authors derived the average density of transmission eigenvalues for a small number of open channels and $\beta=2$ using an auxiliary non-linear sigma model. Their result reads:

$$
\rho_{2}\left(\lambda ; N_{1}, N_{2}<11\right)=\lambda^{\mu} \sum_{n=0}^{N-1}(2 n+\mu+1)\left\{P_{n}^{(\mu, 0)}(1-2 \lambda)\right\}^{2}
$$

where $\mu=\frac{\beta}{2}\left(\left|N_{2}-N_{1}\right|+1\right)-1=\left|N_{2}-N_{1}\right|, N=\min \left(N_{1}, N_{2}\right)$ and $P_{n}^{(\alpha, \beta)}(y)$ is a Jacobi polynomial. 
The authors state in [12]:

...we believe (although we have no formal proof) that Eq. (8) is valid for arbitrary $N_{1}$ and $N_{2}$. This result is consistent with the random-matrix approach of Ref. [3][4], which predicts for the same system a joint distribution of transmission eigenvalues given by the Jacobi ensemble, from which Eq. (8) can be derived. We have thus found independent evidence for the application of the Jacobi ensemble in this problem.

However, the invoked references [3][4] do not mention the Jacobi ensemble, and work out the only case $N_{1}=N_{2}$. More precisely:

1. Ref. [3] reports the jpd (2) restricted to the case $N_{1}=N_{2}$ and $\beta=1,2$. For the case $\beta=2$, the term $\lambda_{i}^{\frac{\beta}{2}\left(\left|N_{2}-N_{1}\right|+1\right)-1}$ in the jpd (2) then disappears, making the use of Legendre polynomials appropriate. For this subcase, the authors derive the average density and the 2-point function, and finally take the large $N_{1}=N_{2}$ limit to get the smoothed macroscopic density $\rho_{2}\left(\lambda ; N_{1}=N_{2} \gg 1\right) \approx N / \pi \sqrt{\lambda(1-\lambda)}$;

2. Ref. [4] deals with all symmetry classes $\beta=1,2,4$ and considers the two cases $N_{1}=N_{2} \gg 1$ or $N_{1}=N_{2}=1$. In the first subcase, the authors derive some quantities of interest with the use of a Coulomb gas approach after the change of variable $\lambda_{i}=1 /\left(1+y_{i}\right), y_{i} \in[0, \infty)$.

We wish to clarify that the average density of transmission eigenvalues for any $N_{1}$ and $N_{2}$ is exactly given by the density of the Jacobi ensemble, where the argument of the Jacobi polynomials is $1-2 \lambda$ (i.e. nothing but $(8)$, for $\beta=2$ ), and this result descends from the application of the standard Orthogonal Polynomial Technique [16][22] to the (modified) jpd (4). In fact, the Jacobi polynomials $P_{n}^{(\mu, 0)}$ appearing in (8) are precisely the orthogonal polynomials over $[-1,1]$ with respect to the weight $(1-y)^{\mu}$ in $(4)$. The cases $\beta=1$ and $\beta=4$ are more complicated, but can be tackled in the same framework (see [23] and references therein). Also, $n$-th order correlation functions can be derived for all three symmetry classes [16][23]. For example, for $\beta=2$ one defines the kernel (see [16], Sections 5.7 and 19.1):

$$
K_{N}(x, y)=x^{\mu / 2} y^{\mu / 2} \sum_{n=0}^{N-1}(2 n+\mu+1) P_{n}^{(\mu, 0)}(1-2 x) P_{n}^{(\mu, 0)}(1-2 y)
$$

and the $n$-th order correlation function is written in terms of the $(n \times n)$ 
determinant:

$$
\begin{aligned}
\rho_{2}\left(\lambda_{1}, \ldots, \lambda_{n}\right) & =\frac{N !}{(N-n) !} \int_{[0,1]^{N-n}} d \lambda_{n+1} \ldots d \lambda_{N} P_{\beta}\left(\lambda_{1}, \ldots, \lambda_{N}\right) \\
& =\operatorname{det}\left[K_{N}\left(\lambda_{j}, \lambda_{k}\right)\right]_{1 \leq j, k \leq n}
\end{aligned}
$$

In particular, the average spectral density (1-point function) is exactly given by:

$$
\rho_{2}\left(\lambda ; N_{1}, N_{2}\right)=\lambda^{\mu} \sum_{n=0}^{N-1}(2 n+\mu+1)\left\{P_{n}^{(\mu, 0)}(1-2 \lambda)\right\}^{2}
$$

extending the result (8) to an arbitrary number of open channels. In Appendix $\mathrm{B}$, we will show that for $\mu \rightarrow 0$ eq. (11) recovers the result by Baranger and Mello [3].

The purpose of this paper is thus twofold:

- having clarified the role of the Jacobi ensemble, and the known results for its spectral density for arbitrary $N_{1}$ and $N_{2}$, we give a closed form expression for moments of the form $\left\langle\lambda_{1}^{m}\right\rangle$ for $m>-\left|N_{2}-N_{1}\right|-1$ and $\beta=2$ through a simple integration over the average density (linear statistic). The formula is exact and non-perturbative, and extends previous results in the literature (Section 2).

- exploiting a less known result by Kaneko, we give an alternative representation for the average density and higher order correlation functions for $\beta=2,4$ in terms of hypergeometric functions of a matrix argument. Thanks to a recent algorithmic progress, this result may prove useful for a numerical implementation which avoids the use of determinants (or quaternion determinants) (Section 3).

\section{A closed form expression for moments}

For simplicity, we consider again the $\beta=2$ case as in [12]. The moments $\left\langle\lambda_{1}^{m}\right\rangle$ can be computed as a simple linear statistic on the transmission eigenvalues:

$$
\left\langle\lambda_{1}^{m}\right\rangle=\int_{0}^{1} d x x^{m} \rho_{2}\left(x ; N_{1}, N_{2}\right)
$$

Known results about $\left\langle\lambda_{1}^{m}\right\rangle$ include:

1. Approximate evaluation for all positive integer $m$ (but valid in the regime $N_{1}, N_{2} \gg 1$ ) [24][25]; also, the generating function for all moments in this limit was first computed in [21]. 
2. Exact evaluation (valid for all $N_{1}, N_{2}, \beta$ ) but only up to $m=4$ (see [20] and references therein).

Assuming $N_{1}<N_{2}$ without loss of generality, we can use (11) and (13) with $N_{1}=N$ and $N_{2}=\mu+N$ :

$$
\left\langle\lambda_{1}^{m}\right\rangle=\sum_{n=0}^{N-1}(2 n+\mu+1) \int_{0}^{1} d x x^{\mu+m} P_{n}^{(\mu, 0)}(1-2 x) P_{n}^{(\mu, 0)}(1-2 x)
$$

After the change of variables $x=(1-t) / 2$ and the definition of Jacobi polynomials as:

$$
P_{n}^{(\mu, 0)}(t)=\frac{1}{n !} \sum_{k=0}^{n} \frac{(-n)_{k}(\mu+n+1)_{k}(\mu+k+1)_{n-k}}{k !}\left(\frac{1-t}{2}\right)^{k}
$$

(where $(a)_{k}=\Gamma(a+k) / \Gamma(a)$ is a Pochhammer symbol), we obtain:

$$
\begin{aligned}
\left\langle\lambda_{1}^{m}\right\rangle & =\frac{1}{2^{\mu+m+1}} \sum_{n=0}^{N-1} \frac{2 n+\mu+1}{n !} \sum_{k=0}^{n} \frac{(-n)_{k}(\mu+n+1)_{k}(\mu+k+1)_{n-k}}{2^{k} k !} \times \\
& \times \int_{-1}^{1} d t(1-t)^{\mu+m+k} P_{n}^{(\mu, 0)}(t)
\end{aligned}
$$

The integral above can be computed for $m>-\mu-1$ ([26], formula 7.391.2) in terms of a hypergeometric function ${ }_{3} F_{2}(-n, \mu+n+1, \mu+m+k+1 ; \mu+$ $1, \mu+m+k+2 ; 1)$. Since the first argument is a negative integer, the series gets truncated to give eventually ${ }^{1}$ :

$$
\left\langle\lambda_{1}^{m}\right\rangle=\sum_{n=0}^{N-1}(2 n+\mu+1) \sum_{k, \ell=0}^{n} \frac{g(k) g(\ell)}{\mu+m+k+\ell+1}
$$

where:

$$
g(\kappa)=(-1)^{\kappa}\left(\begin{array}{l}
n \\
\kappa
\end{array}\right)\left(\begin{array}{c}
n+\mu+\kappa \\
\mu+\kappa
\end{array}\right)
$$

Despite lacking the aesthetic appeal of subcases already considered in the literature [24][20], formula (16) is nevertheless valid for any $\left(N_{1}, N_{2}\right)$ and $m>-\mu-1$, and is fully non-perturbative. After implementing (16) in MATHEMATICA ${ }^{\circledR}$, one can check by direct inspection that:

\footnotetext{
${ }^{1}$ We are grateful to Marcel Novaes for suggesting significant simplifications in (16).
} 
1. the formula (16) agrees with the approximate result in [24] (valid for $\left.N_{1}, N_{2} \gg 1\right)$ :

$$
\left\langle\lambda_{1}^{m}\right\rangle=(\mu+2 N) \sum_{p=1}^{m}\left(\begin{array}{c}
m-1 \\
p-1
\end{array}\right)(-1)^{p-1} c_{p-1}\left(\frac{N(\mu+N)}{(\mu+2 N)^{2}}\right)^{p}
$$

where $c_{p}=\frac{1}{p+1}\left(\begin{array}{c}2 p \\ p\end{array}\right)$ (see Table 1$)$.

2. The shot noise power $\langle P\rangle / P_{0}$, defined as $\left\langle\lambda_{1}\right\rangle-\left\langle\lambda_{1}^{2}\right\rangle$, can be extracted from (16). Thanks to multiple cancellations, the result can be cast in the very simple form:

$$
\frac{\langle P\rangle}{P_{0}}=\frac{N^{2}(\mu+N)^{2}}{(\mu+2 N-1)(\mu+2 N)(\mu+2 N+1)}
$$

which agrees with the known exact result [13][14] (see also eq. (30) below).

3. The average conductance $\langle G\rangle / G_{0}=\left\langle\lambda_{1}\right\rangle$ from (16) can be brought to the simple form:

$$
\frac{\langle G\rangle}{G_{0}}=\frac{N(\mu+N)}{\mu+2 N}
$$

which agrees with the known result [3].

\begin{tabular}{|c|c|c|c|c|}
\hline$\mu$ & $N$ & $\mathrm{~m}$ & Exact (16) & Approximate (18) \\
\hline 4 & 57 & 3 & 18.4240 & 18.4248 \\
4 & 87 & 7 & 18.637 & 18.638 \\
12 & 47 & 19 & 6.7672 & 6.77002 \\
15 & 57 & 29 & 6.67909 & 6.68199 \\
25 & 75 & 59 & 6.34394 & 6.34704 \\
\hline
\end{tabular}

Table 1. Comparison between the moments $\left\langle\lambda_{1}^{m}\right\rangle$ computed by Novaes [24] and our exact derivation (16). Note that the normalization $\int_{0}^{1} d x \rho_{\beta}\left(x ; N_{1}, N_{2}\right)=N_{1}$ implies that the moments are not constrained between 0 and 1 .

\section{A second derivation of the average density and higher-order correlation functions}

In this section, we will derive an alternative expression for the average density of transmission eigenvalues and higher order correlation functions for finite $N_{1}$ and $N_{2}$ and $\beta=2,4$, starting from the jpd (2). Exploiting 
a variant of the Selberg integral evaluated by Kaneko [27], all correlation functions can be expressed in terms of a hypergeometric function of a matrix argument, instead of a determinant of a kernel as in (10) (for $\beta=2$ ).

Consider the joint probability density of transmission eigenvalues (2):

$$
P_{\beta}\left(\lambda_{1}, \ldots, \lambda_{N}\right)=\mathcal{N}_{\beta}^{-1} \prod_{j<k}\left|\lambda_{j}-\lambda_{k}\right|^{\beta} \prod_{i=1}^{N} \lambda_{i}^{\frac{\beta}{2}\left(\left|N_{2}-N_{1}\right|+1\right)-1}, \quad 0 \leq \lambda_{j} \leq 1,
$$

where $N=\min \left(N_{1}, N_{2}\right), \beta=1,2,4$ and the normalization constant is given by (3).

The density of eigenvalues is given by the following multiple integral:

$$
\rho_{\beta}\left(\lambda_{1} ; N_{1}, N_{2}\right)=N \int_{0}^{1} \ldots \int_{0}^{1} d \lambda_{2} \ldots d \lambda_{N} P_{\beta}\left(\lambda_{1}, \ldots, \lambda_{N}\right),
$$

such that the normalization $\int_{0}^{1} \rho_{\beta}\left(x ; N_{1}, N_{2}\right) d x=N$ holds (where again $\left.N=\min \left(N_{1}, N_{2}\right)\right)$.

It turns out that the integral above can be evaluated without the use of the Orthogonal Polynomial technique, which would lead to the formula (11), if one resorts to the following extension of Selberg integral given by Kaneko [27]:

$$
\begin{aligned}
\int_{[0,1]^{n}} & \prod_{j=1}^{n} d x_{j} \prod_{j=1}^{n} x_{j}^{\ell_{1}}\left(1-x_{j}\right)^{\ell_{2}} \prod_{\substack{1 \leq i \leq n \\
1 \leq k \leq m}}\left(x_{i}-t_{k}\right) \prod_{j<k}\left|x_{j}-x_{k}\right|^{\beta}= \\
& C_{12} F_{1}^{(\beta / 2)}\left(-n, \frac{2}{\beta}\left(\ell_{1}+\ell_{2}+m+1\right)+n-1 ; \frac{2}{\beta}\left(\ell_{1}+m\right) ;\left\{t_{1}, \ldots, t_{m}\right\}\right),
\end{aligned}
$$

where $C_{1}$ is a known constant and ${ }_{2} F_{1}^{(\alpha)}$ is a hypergeometric function of a matrix argument. Details about these objects are provided in the appendix.

From (22), one has:

$$
\begin{aligned}
\rho_{\beta}\left(\lambda_{1} ; N_{1}, N_{2}\right) & =\frac{N \lambda_{1}^{\frac{\beta}{2}\left(\left|N_{2}-N_{1}\right|+1\right)-1}}{\mathcal{N}_{\beta}} \int_{[0,1]^{N-1}} d \lambda_{2} \ldots d \lambda_{N} \prod_{j<k}\left|\lambda_{j}-\lambda_{k}\right|^{\beta} \\
& \times \prod_{i=2}^{N} \lambda_{i}^{\frac{\beta}{2}\left(\left|N_{2}-N_{1}\right|+1\right)-1} .
\end{aligned}
$$

Now, the Vandermonde coupling can be decomposed as:

$$
\prod_{j<k}\left|\lambda_{j}-\lambda_{k}\right|^{\beta}=\prod_{j<k, j=2}\left|\lambda_{j}-\lambda_{k}\right|^{\beta} \prod_{j=2}^{N}\left|\lambda_{1}-\lambda_{j}\right|^{\beta},
$$


and, for $\beta=2,4$ the absolute value in all products is immaterial. Hence:

$$
\begin{aligned}
\rho_{\beta}\left(\lambda_{1} ; N_{1}, N_{2}\right) & =\frac{N \lambda_{1}^{\frac{\beta}{2}\left(\left|N_{2}-N_{1}\right|+1\right)-1}}{\mathcal{N}_{\beta}} \int_{[0,1]^{N-1}} d \lambda_{2} \ldots d \lambda_{N} \prod_{j<k, j=2}\left|\lambda_{j}-\lambda_{k}\right|^{\beta} \\
& \times \prod_{i=2}^{N} \lambda_{i}^{\frac{\beta}{2}\left(\left|N_{2}-N_{1}\right|+1\right)-1} \prod_{j=2}^{N}\left(\lambda_{j}-\lambda_{1}\right)^{\beta} .
\end{aligned}
$$

Comparing (26) and (23), after the following identification:

$$
\left\{\begin{aligned}
n & =N-1 \\
\ell_{1} & =\frac{\beta}{2}\left(\left|N_{2}-N_{1}\right|+1\right)-1 \\
\ell_{2} & =0 \\
t_{k} & =\lambda_{1} \quad \forall k=1, \ldots, m \\
m & =\beta
\end{aligned}\right.
$$

one eventually obtains:

$$
\begin{aligned}
& \rho_{\beta}\left(\lambda_{1} ; N_{1}, N_{2}\right)=\frac{N C_{1} \lambda_{1}^{\frac{\beta}{2}\left(\left|N_{2}-N_{1}\right|+1\right)-1}}{\mathcal{N}_{\beta}} \\
& \quad \times{ }_{2} F_{1}^{(\beta / 2)}\left(1-N,\left|N_{2}-N_{1}\right|+N+1 ;\left|N_{2}-N_{1}\right|+3-2 / \beta ; \lambda_{1} \mathbf{1}_{\beta}\right),
\end{aligned}
$$

where we have introduced a customary matrix notation in the last argument of the hypergeometric function. Note that the result (27) is still formally valid for any even integer $\beta$.

We also observe that higher order correlation functions can be easily written down, exploiting the very same eq. (23). For example, the twopoint function $\rho_{\beta}^{(2)}\left(\lambda_{1}, \lambda_{2} ; N_{1}, N_{2}\right)$ can be written (ignoring prefactors) as:

$$
\begin{aligned}
\rho_{\beta}^{(2)}\left(\lambda_{1}, \lambda_{2} ;\right. & \left.N_{1}, N_{2}\right) \propto\left(\lambda_{1} \lambda_{2}\right)^{\frac{\beta}{2}\left(\left|N_{2}-N_{1}\right|+1\right)-1}\left|\lambda_{2}-\lambda_{1}\right|^{\beta} \\
& \times \int_{[0,1]^{N-2}} d \lambda_{3} \ldots d \lambda_{N} \prod_{i=3} \lambda_{i}^{\frac{\beta}{2}\left(\left|N_{2}-N_{1}\right|+1\right)-1} \prod_{j<k, j=3}\left|\lambda_{j}-\lambda_{k}\right|^{\beta} \\
& \times \prod_{j=3}^{N}\left|\lambda_{j}-\lambda_{1}\right|^{\beta} \prod_{j=3}^{N}\left|\lambda_{j}-\lambda_{2}\right|^{\beta}
\end{aligned}
$$

and the $(N-2)$-fold integral is again of the same form as (23) for the 
following values of parameters:

$$
\left\{\begin{array}{rlrl}
n & =N-2 & \\
\ell_{1} & =\frac{\beta}{2}\left(\left|N_{2}-N_{1}\right|+1\right)-1 \\
\ell_{2} & =0 & \\
t_{k} & =\lambda_{1} \quad \forall k=1, \ldots, \beta \\
t_{k} & =\lambda_{2} & \forall k=\beta+1, \ldots, 2 \beta \\
m & =2 \beta &
\end{array}\right.
$$

Hence, this time the matrix argument of the hypergeometric function is $\mathbf{X}^{(2)}:=\operatorname{diag}(\underbrace{\lambda_{1}, \ldots, \lambda_{1}}_{\beta \text { times }}, \underbrace{\lambda_{2}, \ldots, \lambda_{2}}_{\beta \text { times }})$. Note that the 2-point correlation function $\rho_{\beta}^{(2)}\left(\lambda_{1}, \lambda_{2} ; N_{1}, N_{2}\right)$ is manifestly symmetric in the two arguments as it should, due to the symmetry of Jack polynomials (see appendix). It is worth mentioning that higher order correlation functions can be written down easily along the same lines.

Thanks to a very efficient MATLAB ${ }^{\circledR}$ implementation of this kind of hypergeometric functions by Koev and Edelman [28], the density itself, linear statistics (one-dimensional integrals over the density) and $n$-th order correlation functions can be numerically tackled very easily. In particular, these results entirely avoid the use of (quaternion) determinants and (skew-)orthogonal polynomials which would arise from the canonical RMT treatment and can get computationally demanding for high $N_{1,2}$ and $n$. Conversely, the computational complexity of the algorithm in [28] is only linear in the size of the matrix argument $(\beta n)$.

In the following, we shall provide some plots of the spectral density for different numbers of incoming and outgoing leads, and $\beta=2$ (Fig. 1). The agreement between the two alternative formulas (11) and (27) is excellent.

As a final check, we also numerically compute the prototype of linear statistics, i.e. the (normalized) average shot noise power $\langle P\rangle / P_{0}$ (see (1)), defined as:

$$
\langle P\rangle / P_{0}=\int_{0}^{1} d x \rho_{\beta}\left(x ; N_{1}, N_{2}\right) x(1-x)
$$

where $\rho_{\beta}\left(x ; N_{1}, N_{2}\right)$ is taken from (27). The result has to agree with the analytical expression [13][14]:

$$
\frac{\langle P\rangle}{P_{0}}=\frac{N_{1}\left(N_{1}-1+2 / \beta\right) N_{2}\left(N_{2}-1+2 / \beta\right)}{(\tilde{N}-2+2 / \beta)(\tilde{N}-1+2 / \beta)(\tilde{N}-1+4 / \beta)}
$$

where $\tilde{N}=N_{1}+N_{2}$. We compare in Table 2 the theoretical result (30) with the numerical integration of $(29)$, obtained in MATLAB ${ }^{\circledR}$ with a standard 
integration routine. The agreement we found is excellent, thus confirming the correctness of $(27)$.

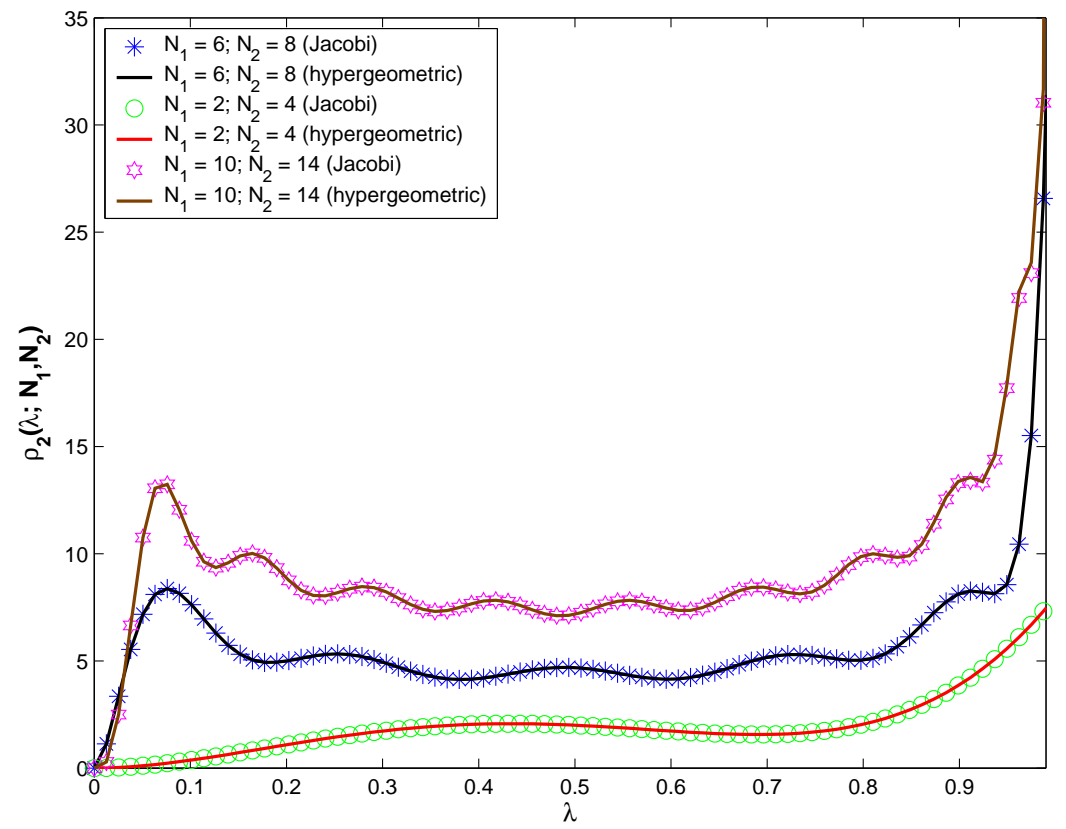

Fig. 1. Density of transmission eigenvalues for $\beta=2$ and different values for the pair $\left(N_{1}, N_{2}\right)$. The plot symbols are used for RMT formula (11), whereas solid lines represent the alternative formula (27).

\begin{tabular}{|c|c|c|c|c|}
\hline$N_{1}$ & $N_{2}$ & $\beta$ & Theory & Numerical \\
\hline 4 & 7 & 2 & 0.5939393 & 0.5939393 \\
8 & 11 & 2 & 1.1321637 & 1.1321639 \\
3 & 9 & 2 & 0.4248251 & 0.4248251 \\
4 & 7 & 4 & 0.5805422 & 0.5805424 \\
3 & 5 & 4 & 0.4326923 & 0.4326923 \\
\hline
\end{tabular}

Table 2. Comparison between the theoretical expression for the average shot-noise power (30) and the numerical integration of (29), for different values of $N_{1}, N_{2}$ and $\beta$. 


\section{Conclusions}

We have clarified that the average density of transmission eigenvalues for chaotic cavities is analytically known from the Jacobi ensemble of random matrices, as well as all higher-order correlation functions. The known formula for the average density coincides with the one derived with a mapping to a non-linear sigma model by Araújo and Macêdo, but is rigorously valid for any number of open channels. With an elementary integration over this density, we derived a general formula for the moments $\left\langle\lambda_{1}^{m}\right\rangle$, which is non-perturbative and valid for arbitrary large $m$ and $\beta=2$. Also, a second derivation is offered for the spectral density and higher order correlation functions, which does not make use of orthogonal polynomials or determinantal structures. Thanks to a recent algorithmic progress, this result, exploiting a hypergeometric function of a matrix argument, may be numerically easier to implement than high-order (quaternion) determinants. All

the results are consistent with numerical checks and known formulas in the literature.

\section{Acknowledgments}

PV has been supported by a Marie Curie Early Stage Training Fellowship (NET-ACE project). We are indebted with Gernot Akemann and Dmitry Savin for clarifying discussions and useful suggestions. We are grateful to Piet Brouwer, Marcel Novaes and Victor A. Gopar for pointing out relevant references. We also thank Satya N. Majumdar and Oriol Bohigas for collaboration on related topics and many helpful advices.

\section{Appendix A}

Hypergeometric function of a matrix argument

Following Kaneko [27], we first report the definition of the constant $C_{1}$ appearing in (23):

$$
C_{1}:=S_{n, 0}\left(\ell_{1}+m, \ell_{2}, \beta\right)
$$

where:

$$
S_{n, 0}\left(y_{1}, y_{2}, z\right):=\prod_{i=1}^{n} \frac{\Gamma\left(i \frac{z}{2}+1\right) \Gamma\left(y_{1}+1+(i-1) \frac{z}{2}\right) \Gamma\left(y_{2}+1+(i-1) \frac{z}{2}\right)}{\Gamma\left(\frac{z}{2}+1\right) \Gamma\left(y_{1}+y_{2}+2+(n+i-2) \frac{z}{2}\right)} .
$$

The hypergeometric function of a matrix argument [29] takes a symmetric matrix $(m \times m) \mathbf{X}$ as input and provides a real number as output. It is defined as a series of Jack functions of parameter $\beta$, which generalize 
the Schur function, the zonal polynomial and the quaternion zonal polynomial to which they reduce for $\beta=1,2,4$ respectively. Given a partition $\kappa$ of an integer $k$, i.e. a set of integers $\kappa_{1} \geq \kappa_{2} \geq \ldots \geq 0$ such that $|\kappa|=\kappa_{1}+\kappa_{2}+\ldots=k$, and a matrix $\mathbf{X}$, the Jack function $C_{\kappa}^{(\beta)}(\mathbf{X})$ is a symmetric and homogeneous polynomial of degree $|\kappa|$ in the eigenvalues $x_{1}, \ldots, x_{m}$ of $\mathbf{X}$.

The hypergeometric function is defined as:

$$
{ }_{p} F_{q}^{(\beta)}\left(a_{1}, \ldots, a_{p} ; b_{1}, \ldots, b_{q} ; \mathbf{X}\right):=\sum_{k=0}^{\infty} \sum_{\kappa \vdash k} \frac{\left(a_{1}\right)_{\kappa}^{(\beta)} \ldots\left(a_{p}\right)_{\kappa}^{(\beta)}}{k !\left(b_{1}\right)_{\kappa}^{(\beta)} \ldots\left(b_{q}\right)_{\kappa}^{(\beta)}} C_{\kappa}^{(\beta)}(\mathbf{X}),
$$

where the symbol $\kappa \vdash k$ means that $\kappa$ is a partition of $k$ and $(a)_{\kappa}^{(\beta)}=$ $\prod_{(i, j) \in \kappa}\left(a-\frac{i-1}{\beta}+j-1\right)$ is a generalized Pochhammer symbol.

The series (A.3) converges for any $\mathbf{X}$ if $p \leq q$; it converges if $\max _{i}\left|x_{i}\right|<1$ and $p=q+1$; and diverges if $p>q+1$, unless it terminates [28][29][18].

\section{Appendix B}

The $\mu \rightarrow 0$ limit of the spectral density.

In the case $N_{1}=N_{2}=N$ and $\beta=2$, the average spectral density was computed exactly in [3] as:

$$
\rho_{2}\left(\lambda ; N_{1}=N_{2}=N\right)=\frac{N^{2}}{4 \lambda(1-\lambda)}\left\{P_{N}^{2}(\alpha)-2 \alpha P_{N}(\alpha) P_{N-1}(\alpha)+P_{N-1}^{2}(\alpha)\right\}
$$

where $\alpha=2 \lambda-1$ and $P_{N}(x)$ is a Legendre polynomial.

This case corresponds to $\mu \rightarrow 0$ in eq. (11). In this appendix, we show explicitly how to get from (11) to (B.1).

First, we remark that the identity between Jacobi and Legendre polynomials $P_{n}^{(0,0)}(x)=P_{n}(x)$ holds [formula 22.5.35 in [30]]. Hence, in the case $\mu \rightarrow 0$ we have from (11):

$$
\rho_{2}\left(\lambda ; N_{1}=N_{2}=N\right)=\sum_{n=0}^{N-1}(2 n+1)\left\{P_{n}(1-2 \lambda)\right\}^{2}
$$

Next, we use the Christoffel-Darboux formula for Legendre polynomials [see formula 22.12.1 in [30]] at equal arguments $x=y=1-2 \lambda$ :

$$
\sum_{n=0}^{N-1}(2 n+1) P_{n}^{2}(y)=N\left[P_{N}^{\prime}(y) P_{N-1}(y)-P_{N-1}^{\prime}(y) P_{N}(y)\right]
$$


Then, we exploit the differential relation [22.8.5 in [30]]:

$$
\left(1-y^{2}\right) P_{n}^{\prime}(y)=-n y P_{n}(y)+n P_{n-1}(y)
$$

to get:

$$
\begin{aligned}
\rho_{2}\left(\lambda ; N_{1}=N_{2}=N\right) & =\frac{N}{1-y^{2}}\left[N P_{N-1}^{2}(y)-y P_{N}(y) P_{N-1}(y)-(N-1) \times\right. \\
& \left.\times P_{N}(y) P_{N-2}(y)\right]\left.\right|_{y=1-2 \lambda}
\end{aligned}
$$

Thanks to the recurrence relation [22.7.10 in [30]], we obtain the following identity for $P_{N-2}(y)$ :

$$
P_{N-2}(y)=\frac{1}{N-1}\left[(2 N-1) y P_{N-1}(y)-N P_{N}(y)\right]
$$

which is then substituted into (B.5). Eventually, given that $y=1-2 \lambda$ and the Legendre polynomials have the same parity of their index, we obtain:

$$
\begin{aligned}
\rho_{2}\left(\lambda ; N_{1}=N_{2}=N\right) & =\frac{N^{2}}{4 \lambda(1-\lambda)}\left[P_{N}^{2}(2 \lambda-1)-2(2 \lambda-1) P_{N}(2 \lambda-1) \times\right. \\
& \left.\times P_{N-1}(2 \lambda-1)+P_{N-1}^{2}(2 \lambda-1)\right]
\end{aligned}
$$

in complete agreement with (B.1).

\section{REFERENCES}

[1] Ya.M. Blanter and M. Büttiker, Phys. Rep. 336, 1 (2000). [cond-mat/9910158]

[2] C.W.J. Beenakker, Rev. Mod. Phys. 69, 731 (1997). [cond-mat/9612179]

[3] H.U. Baranger and P.A. Mello, Phys. Rev. Lett. 73, 142 (1994). [cond-mat/9403056]

[4] R.A. Jalabert, J.-L. Pichard and C.W.J. Beenakker, Europhys. Lett. 27, 255 (1994). [cond-mat/9403073]

[5] M. Büttiker, Phys. Rev. Lett. 65, 2901 (1990).

[6] V.A. Khlus, Soviet Physics - JETP 66, 1243 (1987).

[7] G.B. Lesovik, JETP Lett. 49, 592 (1989).

[8] Yu.V. Nazarov, in Quantum Dynamics of Submicron Structures, edited by H.A. Cerdeira, B. Kramer and G. Schön (Kluwer, Dordrecht, 1995), NATO ASI Series E: Applied Science, Vol. 291, p. 687.

[9] H. Schanz, M. Puhlmann and T. Geisel, Phys. Rev. Lett. 91, 134101 (2003). [cond-mat/0304265] 
[10] R.S. Whitney and Ph. Jacquod, Phys. Rev. Lett. 96, 206804 (2006). [cond-mat/0512516]

[11] M.H. Pedersen, S.A. van Langen and M. Büttiker, Phys. Rev. B 57, 1838 (1998). [cond-mat/9707086]

[12] J.E.F. Araújo and A.M.S. Macêdo, Phys. Rev. B 58, R13379 (1998).

[13] P. Braun, S. Heusler, S. Müller and F. Haake, J. Phys. A: Math. Gen. 39, L159 (2006). [cond-mat/0511292]

[14] D.V. Savin and H.-J. Sommers, Phys. Rev. B 73, 081307(R) (2006). [cond-mat/0512620]

[15] E.N. Bulgakov, V.A. Gopar, P.A. Mello and I. Rotter, Phys. Rev. B 73, 155302 (2006). [cond-mat/0511424]

[16] M.L. Mehta, Random Matrices, 3rd Edition, (Elsevier-Academic Press, Amsterdam, 2004).

[17] P.J. Forrester, J. Phys. A: Math. Gen. 39, 6861 (2006). [math-ph/0601024]

[18] P.J. Forrester, Log-gases and random matrices, http://www.ms.unimelb.edu.au/ matpjf/matpjf.html.

[19] K. Richter and M. Sieber, Phys. Rev. Lett. 89, 206801 (2002). [cond-mat/0205158]

[20] D.V. Savin, H.-J. Sommers and W. Wieczorek, [arXiv:0711.1764] (2007).

[21] P.W. Brouwer and C.W.J. Beenakker, J. Math. Phys. 37, 4904 (1996). [cond-mat/9604059]

[22] T. Nagao and M. Wadati, Journal of the Physical Society of Japan 60, 3298 (1991).

[23] S. Ghosh, [arXiv:0711.4432] (2007).

[24] M. Novaes, Phys. Rev. B 75, 073304 (2007). [cond-mat/0701141]

[25] G. Berkolaiko, J.M. Harrison and M. Novaes, [cond-mat/0703803] (2007).

[26] I.S. Gradshteyn and I.M. Ryzhik, Table of Integrals, Series, and Products, ed. Alan Jeffrey and Daniel Zwillinger, 7th edition (2007).

[27] J. Kaneko, SIAM J. Math. Anal. 24, 1086 (1993).

[28] P. Koev and A. Edelman, Mathematics of Computation 75, 833 (2006). [math/0505344]

[29] R. J. Muirhead, Aspects of multivariate statistical theory (John Wiley \& Sons Inc., New York, 1982).

[30] M. Abramowitz and I.A. Stegun, Handbook of Mathematical Functions (Dover Publications,Inc.,New York, 1972). 